\title{
The Longitudinal Association Between Preadolescent Facial Emotion Identification and Family Factors, and Psychotic Experiences in Adolescence (The TRAILS Study)
}

\author{
Laura A. Steenhuis ${ }^{1}$ (D) . Gerdina H.M. Pijnenborg ${ }^{1,2}$. Elisabeth C.D. van der Stouwe ${ }^{3,5} \cdot$ Catharina A. Hartman $^{4}$. \\ André Aleman $^{1,3} \cdot$ Agna A. Bartels-Velthuis ${ }^{5} \cdot$ Maaike H. Nauta ${ }^{1}$
}

Published online: 4 September 2019

(c) The Author(s) 2019

\begin{abstract}
The current study examines whether facial emotion identification and family factors at preadolescence (age 11) predict psychotic experiences 5 years later during adolescence (age 16) and whether family factors may mediate the association between facial emotion identification and psychotic experiences. Data was obtained from the epidemiological cohort TRAILS $(\mathrm{N}=2059)$. At preadolescence, a facial emotion identification test and three questionnaires to assess family functioning, perceived parenting styles and parenting stress, were administered. At adolescence, a questionnaire on psychotic experiences was administered. Facial emotion identification at preadolescence was not associated with psychotic experiences at adolescence, and the mediational role of family functioning was not further explored. However, increased overprotective parenting at preadolescence was associated with a higher frequency of psychotic experiences and delusions at adolescence. Future research may examine the mechanism behind the role of overprotective parenting on psychotic experiences during adolescence.
\end{abstract}

Keywords Adolescence $\cdot$ Psychosis $\cdot$ Parenting $\cdot$ Family functioning $\cdot$ Social cognition

Laura A. Steenhuis

1.a.steenhuis@rug.nl

1 Department of Clinical Psychology and Experimental Psychopathology, Faculty of Behavioural and Social Sciences, University of Groningen, Grote Kruisstraat 2/1, 9712 TS Groningen, The Netherlands

2 Department of Psychotic Disorders, GGZ Drenthe, Assen, The Netherlands

3 Department of Neuroscience, University Medical Center Groningen, University of Groningen, Groningen, The Netherlands

4 Interdisciplinary Center Psychopathology and Emotion Regulation, Department of Psychiatry, University Medical Center Groningen, University of Groningen, Groningen, The Netherlands

5 Rob Giel Research Center, University Center for Psychiatry, University Medical Center Groningen, University of Groningen, Groningen, The Netherlands

\section{Introduction}

Psychotic disorders have often been associated with social cognitive impairments [1]. One of the domains of social cognition is facial emotion identification [2], which refers to the ability to accurately identify emotional expressions from another person's face. The 'basic' set of emotions (anger, disgust, fear, sadness, surprise and happiness) as proposed by Ekman and colleagues are each characterized by a distinct facial expression, physiology and evolutionary purpose [3]. The ability to accurately recognize these emotions is crucial in facilitating emotional connections and communicating effectively with others. In psychotic disorders, recognition of positive expressions (happiness) is preserved and recognition of negative expressions (anger, fear, sadness and disgust) is impaired [4-7], although some studies report impairments for both positive and negative emotions [8].

Recent studies demonstrated that impairments in the identification of facial affect are not only found in chronic psychosis [9], but also in first episode psychosis [10], the ultra-high risk phase of psychosis [11, 12], and in siblings [13]. The evidence suggests that early impairment may show 
up for specific emotions, rather than as a general deficit [10]. Deficits in facial emotion identification have been hypothesized to play a role in the development of psychotic experiences. To specify, facial emotion identification deficits could give rise to paranoia (an inability to understand others could feed negative interpretations [5, 14]), delusions (an inability to correct faulty interpretations can cause and support delusional ideation [15]), and potentially hallucinations (continuous erroneous interpretation of social situations and others can lead to social stress, hyper vigilance, and hallucinatory experiences [16] (see also a review by [17]). Overall, an impairment in facial emotion identification may be a trait vulnerability for psychosis, rather than a consequence of the disorder. It is important to investigate at which point facial emotion identification impairments can be 'detected' as to examine when early interventions may be possible and effective. Given that psychotic experiences are prevalent in samples of youth [18-20], and may signify a precursor to psychotic disorders [21], it is fruitful to examine whether reduced facial emotion identification in preadolescence is associated with psychotic experiences during adolescence.

Social cognitive impairments have been found to contribute to diminished social functioning in psychotic disorders [22]. If deficits in facial emotion identification are present from childhood, this may already lead to problems in the development of socially competent behaviors and interactions. Given the importance of the family environment for children and adolescents' functioning [23], it is possible that children with poor facial emotion identification skills have more difficulty functioning in the family environment as well. For example, children with poor facial emotion identification skills may perceive parenting as more negative, either due to inaccurate identification of emotions of their parents, or due to an accurate perception of more rejective or overprotective parenting as a reaction to their lower social cognitive abilities. Therefore, if facial emotion identification abilities predict psychotic experiences throughout adolescence, it may be especially interesting to explore the possibility whether this association might be mediated, at least partially, by family functioning.

The family context has gained much attention in psychosis studies, mostly in more acute and chronic phases of illness [24-30]. There is a strong indication that family factors such as expressed emotion [24, 27], the family rearing environment $[25,28]$ and family communication $[26,30]$ are important predictors of the prognosis of psychosis once an individual has transitioned to a first psychotic episode. Several prospective studies have found that patients with family members who are high in expressed emotion (overinvolvement, high criticism, and negative affective style) are at an increased risk of relapse in schizophrenia over a period of nine to 12 months $[24,31]$. Also in children with elevated mental health problems, parental styles (such as communication deviance, expressed emotion or affective style), significantly predicted schizophrenia spectrum disorders in adulthood in a 15 year prospective longitudinal study [26], although it is important to note that this sample was limited in its size $(n=50)$. In the ultra-high risk phase of psychosis (before the first psychotic episode), family functioning (a positive warm environment) has been shown to be protective $[32,33]$, both for reducing negative and disorganized symptoms, and improvement in functioning over a period of 3 [32] and 6 [33] months. Whether the family environment and parenting styles are predictive of the development and course of psychotic experiences (rather than a reaction towards clinical symptoms) during adolescence, remains understudied so far.

The aim of the current study is to examine whether a) facial emotion identification and b) family factors at preadolescence (age 11) predict psychotic experiences 5 years later during adolescence (age 16). We expect that both lower facial emotion identification abilities and more negative family functioning in preadolescence will predict a higher frequency of psychotic experiences at adolescence. If confirmed that facial emotion identification abilities are associated with psychotic experiences in adolescence, we will further explore whether functioning in the family environment (at least partially) mediates the relationship between facial emotion identification and psychotic experiences. Given that childhood mental health is associated with parenting behaviors at preadolescence [34] and is likely to predict adult mental health, the current study will control for pre-adolescent mental health problems (internalizing and externalizing behaviors).

\section{Methods}

\section{Sample}

Data used in the current study were collected as part of the longitudinal 'TRacking Adolescents Individual Lives Survey' (TRAILS), a prospective cohort study which aims to elucidate the etiology of mental health problems during adolescence [35, 36]. The National Dutch Medical Ethical Committee approved this study and the research has been performed in accordance with the ethical standards laid down in the 1964 Declaration of Helsinki and its later amendments. Written informed consent was obtained from all adolescents and their parents in this study. As done in previous studies in this cohort [37], we merged data from two TRAILS samples, a large population-based birth cohort $(n=2230)$ and a smaller parallel clinic-referred cohort $(n=543)$, in order to acquire a large sample with a wide variation in mental health. Data of the first and third data collection waves with mean ages of around 11 (T1) and 16 (T3) years were used 
for the current study. Participants were included if they at least completed the assessment on psychotic experiences at T3 $(n=2059)$. Due to missing data, $N$ varies between 1956 and 2059 in the total sample.

Full details on the sampling procedure, descriptive statistics, response rates and selective attrition have all been provided in previous studies [36, 38]. In summary, to obtain the population cohort, TRAILS approached 135 primary schools in five municipalities in the north of the Netherlands, of which $90.4 \%$ agreed to participate. After contacting eligible preadolescents and their parents, 2230 participants (76\% of those that were contacted) were enrolled in the study at $\mathrm{T} 1$ (mean age $=11.1$ years, $\mathrm{SD}=.0 .56 ; 49.2 \%$ boys). Five years later, $81 \%$ of them participated at $\mathrm{T} 3(\mathrm{~N}=1816$; mean age, 16.3 years, $\mathrm{SD}=.0 .7 ; 48 \%$ boys). The two data waves included in this study ran from March 2001 to July 2002 (T1), and from September 2005 to August 2007 (T3). The clinic-referred cohort contained preadolescents who had been referred to the Groningen University Child and Adolescent Psychiatric Outpatient Clinic at any point in their life. At T1, 543 participants ( $43 \%$ of those that were contacted) participated in the study (mean age $=11.1$ years, $\mathrm{SD}=0.50$; $65.9 \%$ boys). In total 416 (76.6\%) of them completed measurements at T3. The data waves in the clinic-referred cohort started 2 years after the population cohort: from September 2004 to December 2005 (T1), and from and September 2009 to February 2011 (T3). The same design and instruments were used for both cohorts.

\section{Measures}

To assess facial emotion identification at T1, we used the Identification of Facial Expressions Task, which is part of the Amsterdam Neuropsychological Tasks program (ANT [39]). This task is a reliable and valid instrument with acceptable test-retest reliability, and construct, criterion, and discriminant validity [39-41]. Trained undergraduate psychologists assessed each participant individually. The task consists of six parts of 40 trials each, divided over 20 target and 20 non-target trails. Each part focusses on a specific emotion (happy, sad, angry, fear, surprise and disgust) and lasts $5 \mathrm{~min}$ in total. Participants were instructed to press the yes-button for a target emotion and the no-button if a different emotion was displayed. For our study, we selected all emotions except surprise, as we focused explicitly on positive and negative emotions and surprise is considered as neither positive nor negative [42]. Each emotion was examined separately, as early impairment may show up for specific emotions, rather than a general deficit [10]. For each emotion the error proportion (EP) and reaction time (RT) was calculated. EPs were calculated as the mean proportion of misses and false alarms, using the subsequent equation: $\mathrm{EP}=(($ misses $/($ misses + hits $))+($ false alarms $/$ (false alarms + correct rejections)))/2. RTs were calculated by the mean RT across hits and correct rejections. EPs and RTs that were more than four standard deviations above the mean [43] as well as participants performing at chance level of accuracy (50\% or more errors) were considered missing [44]. In addition, outliers in one outcome parameter were also noted missing for the other, as EP and RT may influence each other. For each emotion, standardized Z-scores were created for both the RTs and EPs. It is important to examine both the EPs and RTs of emotions, as both aspects could reveal distinct and independent associations with the development of psychotic experiences [45]. Therefore, 10 variables of facial emotion identification were constructed: EP happy, EP sad, EP angry, EP fear, EP disgust, RT happy, RT sad, RT angry, RT fear and RT disgust.

To assess family functioning at T1, a modified version of the General Functioning Scale of the McMaster Family Assessment Device (FAD; [46]) was administered to the primary parent. The FAD has shown to have adequate test-retest reliability, good divergent and convergent validity, in addition to adequate sensitivity and specificity [47]. The scale includes six dimensions of family functioning, consisting of communication, problem solving, affective responsiveness, affective involvement, roles and behavior control. The scale comprises twelve items with a 4-point scale, ranging from 1 (totally disagree) to 4 (totally agree). A sum score was computed by adding up all items (a higher score indicates lower family functioning).

To assess perceived parenting style at T1, the EMBUC [48] was administered, which is the child version of the EMBU (English translation: My Memories of Upbringing; [49]). The EMBU-C has good psychometric properties and convergent validity [48]. The questionnaire contains the following three scales: Rejection (12 items), Emotional Warmth (18 items), and Overprotection (12 items). Items are assessed using a 4-point scale, ranging from 0 (no, never) to 4 (yes, almost always). Responses of fathers and mothers were highly correlated for rejection $(r=0.68, p<0.001)$, emotional warmth $(r=0.79, p<0.001)$ and overprotection $(r=0.81, p<0.001)$, and therefore, in line with previous TRAILS papers [34, 50-52], scores were combined (averaged) for both parents. If information for only one parent was present, the score for the one parent was used.

To assess parental stress at T1, a short Dutch form of the Parental Stress Index (PSI [53]) was administered. The Dutch version has been found to have good psychometric properties and construct validity [54]. It is a 25 -item questionnaire to assess the magnitude of stress in the parent-child relationship. Items are rated by the parent on a 6-point scale from 1 (disagree very much) to 6 (agree very much). The instrument contains two subscales, assessing the child's characteristics (11 items) and the parents' characteristics in the parenting context (14 items). A previous 
study [55] conducted a factor analysis of this measure in the current TRAILS sample, and discovered that one item did not load on either the child or the parent factor (item 24: "I feel confident about the future upbringing of my child"). Therefore, this item was excluded in the TRAILS cohort. For the purpose of this study, only the parent subscale was used to obtain a measure of perceived stress for the parent.

To assess childhood mental health at T1, the Youth SelfReport (YSR [56]) was administered. The YSR has a good test-retest reliability and discriminative validity [56]. In this 112-item questionnaire, descriptions of emotions and behaviors are rated on a three-point scale (not true (0), somewhat or sometimes true (1) and very often true (2)). These items assess two broad dimensions of behavior problems: internalizing (anxious/depressed, withdrawn/depressed and somatic complaints) and externalizing (aggressive behavior and rulebreaking behavior) problems. For the current study, a total score of all problem behaviors was computed based on 105 items (in line with [57]).

To assess psychotic experiences at $\mathrm{T} 3$, the Community Assessment of Psychic Experiences CAPE [58, 59] was used. The CAPE is a self-report questionnaire with good psychometric properties, discriminative validity [60] and test-retest reliability [58]. The positive experiences subscale has 20 items assessing the frequency and distress of positive experiences (e.g. delusions and hallucinations) separately. The frequency/distress of each item is assessed on a four-point scale [(1) never/no distress, (2) sometimes/a bit distressed, (3) often/quite distressed, and (4) nearly always/ very distressed). For the current study, the frequency of positive experiences was used. Based on a factor analysis [61]] a previous study found five underlying dimensions of the CAPE that are differently associated with risk of future psychopathology. Their study [61] demonstrated that hallucinations, delusions and paranoia, but not grandiosity and paranormal beliefs, were mostly associated with distress and future psychopathology. For the current study these three risk sub-domains were separately identified by calculating a sum score of delusions (8 items) and paranoia (5 items), and a categorical score of hallucinations as either absent or present $(0 / 1)$. Given the low endorsement rate of hallucinations in this sample, adolescents received a 'present' score on the hallucination variable if they endorsed at least one (or more) of the three hallucination items.

\section{Statistical Analyses}

Analyses were carried out in SPSS 25. To examine whether the hypothesized predictors were related to the outcomes of our study, Pearson's correlations were first computed between facial emotion identification variables (RTs, EPs), psychotic experiences (total frequency, hallucinations, delusions and paranoia), and family factors (family functioning, overprotective, warm and rejective parenting, and parental stress). With the relevant associations identified, a number of multiple linear and logistic regression models were run to examine our hypotheses in a step-wise approach. All assumptions of these analyses (e.g. homoscedasticity and normality of residuals) were checked beforehand. First, psychotic experiences (age 16) were predicted by facial emotion identification variables (age 11) (linear and logistic regression models). Second, psychotic experiences (age 16) were predicted by family factors at preadolescence (age 11) (linear and logistic regression models). Third, family factors were predicted by facial emotion identification (both at age 11) (linear regression models). Findings were corrected for multiple testing with the Bonferroni-Holmes correction, thus correcting the $p$ value per step off, starting with the lowest $p$ value [62]. All analyses were controlled for age, sex and pre-adolescent mental health problems. If our first hypothesis was met, we aimed to explore whether family functioning (age 11) mediates the relationship between the relevant facial emotion identification variable (age 11) and psychotic experiences (age 16). This was done with the computational process PROCESS [63], for which a 'parallel multiple mediation model' was computed, where $\mathrm{X}$ (the causal variable: facial emotion perception), was modeled to influence $Y$ (the outcome variable: psychotic experiences) directly, as well as indirectly, through multiple mediator variables (the mediators: family functioning, overprotective, warm and rejective parenting, and parental stress).

\section{Results}

\section{Descriptives}

Characteristics of the sample and assessments outcomes are given in Table 1. In the identification of facial emotions task, positive emotions were easier to recognize than negative emotions, as denoted by lower reaction times $(\mathrm{t}(2641)=76.32, \mathrm{p}<0.01)$ and lower proportion of errors $(\mathrm{t}(2641)=57.29, \mathrm{p}<0.01)$. In Table 2 , correlations between all variables are displayed.

\section{Associations Between Facial Emotion Identification Abilities at Preadolescence (Age 11) and Psychotic Experiences at Adolescence (Age 16)}

Facial emotion identification abilities at age 11 were not significantly associated with delusions at age 16 , and thus not further examined in the regression models (see Table 2). Table 3 demonstrates both linear and logistic regression models, in which frequency of psychotic experiences, hallucinations and paranoia is predicted by facial emotion identification (EPs and RTs), after adjustment for confounders. 
Table 1 Characteristics of the sample and assessments outcomes

\begin{tabular}{|c|c|c|c|}
\hline & $\mathrm{N}$ & $\begin{array}{l}\text { Mean }(\mathrm{SD}) / \\
\text { frequency } \\
(\%)\end{array}$ & Range \\
\hline Age & 2059 & $16.17(0.69)$ & $14.42-18.36$ \\
\hline Sex $(\%$ female $)$ & 2059 & $1018(49.4)$ & \\
\hline \multicolumn{4}{|l|}{$\mathrm{T} 1$} \\
\hline $\begin{array}{l}\text { Mental health (YSR; total prob- } \\
\text { lems) }\end{array}$ & 2018 & $0.36(0.19)$ & $0.00-1.18$ \\
\hline \multicolumn{4}{|c|}{ Facial emotion recognition (IFE task) } \\
\hline \multicolumn{4}{|l|}{ EP } \\
\hline Happy & 2030 & $3.31(3.45)$ & $0-17.50$ \\
\hline Sad & 2033 & $12.88(9.41)$ & $0-45$ \\
\hline Angry & 2020 & $8.34(6.18)$ & $0-35$ \\
\hline Fear & 2020 & $7.74(6.84)$ & $0-37.50$ \\
\hline Disgust & 2026 & $6.16(5.53)$ & $0-30$ \\
\hline \multicolumn{4}{|l|}{ RT } \\
\hline Happy & 2030 & $880(206)$ & $458-1750$ \\
\hline $\mathrm{Sad}$ & 2033 & $1210(286)$ & $528-2449$ \\
\hline Angry & 2020 & $1116(257)$ & $581-2188$ \\
\hline Fear & 2020 & $1113(280)$ & $552-2323$ \\
\hline Disgust & 2026 & $1062(250)$ & 546-2091 \\
\hline Family functioning (FAD) & 1956 & $1.79(0.38)$ & $1.00-4.00$ \\
\hline Parental stress (PSI) & 1959 & $1.93(0.89)$ & $1.00-5.60$ \\
\hline \multicolumn{4}{|l|}{ Parenting behavior (EMBU-C) } \\
\hline Warm parenting & 2047 & $3.22(0.49)$ & $1.17-4.00$ \\
\hline Rejective parenting & 2046 & $1.51(0.32)$ & $1.00-3.44$ \\
\hline Overprotective parenting & 2046 & $1.86(0.37)$ & $1.00-3.44$ \\
\hline \multicolumn{4}{|l|}{$\mathrm{T} 3$} \\
\hline \multicolumn{4}{|l|}{ Psychotic experiences (CAPE) } \\
\hline Total frequency & 2059 & $1.28(0.23)$ & $1.00-2.85$ \\
\hline Hallucinations $(\mathrm{N}, \%)$ & 2051 & $299(14.6)$ & \\
\hline Delusions & 2037 & $1.20(1.73)$ & $0.00-17.00$ \\
\hline Paranoia & 2039 & $2.61(1.77)$ & $0.00-10.00$ \\
\hline
\end{tabular}

T1 Age 11, T3 Age 16

$I F E$ identification of facial expressions task, $E P$ error percentage (raw), $R T$ reaction time (raw), FAD family assessment device, $P S I$ parental stress index, $E M B U-C$ my memories of upbringing, $C A P E$ community assessment of psychic experiences

The results demonstrate that facial emotion identification abilities at age 11 were not significantly associated with psychotic experiences at age 16 . In the absence of an association, mediation by family factors was not explored.

\section{Associations Between Family Factors at Preadolescence (Age 11) and Psychotic Experiences at Adolescence (Age 16)}

Table 4 shows the results from four regression models (both linear and logistic) predicting psychotic experiences (frequency, hallucinations, delusions and paranoia) with family factors, after correcting for confounders. Findings demonstrate that overprotective parenting at age 11 was positively associated with both the frequency of psychotic experiences and delusions at age 16 .

\section{Associations Between Facial Emotion Identification Abilities and Family Factors at Preadolescence (Age 11)}

Overprotective parenting was not significantly associated with family factors at age 11, and thus not further examined in the regression models (see Table 2). Table 5 shows the results from four linear regression models, predicting family factors (family functioning, parental stress, warm and rejective parenting) by facial emotion perception (EPs and RTs), after correcting for confounders. The results demonstrate that facial emotion perception abilities were not significantly associated with family factors at age 11 .

\section{Post-hoc Exploration: The 5\% Lowest Scores on Facial Emotion Identification Abilities and the Frequency of Psychotic Experiences}

We hypothesized that perhaps only adolescents who scored very poorly on facial emotion identification at preadolescence were more vulnerable for developing psychotic experiences at adolescence. Therefore, to investigate whether a specific subsample, namely preadolescents with the lowest scores (highest 5\% of EPs and longest 5\% of RTs) on the facial emotion identification task are at an increased risk for psychotic experiences in adolescence, we conducted a post hoc exploration. The group of 5\% lowest scorers on the emotion perception task had an average EP (\%) of 13.67 (SD: 1.64), 38.46 (SD: 3.42), 24.00 (SD: 4.59), 27.20 (SD: 4.76) and 20.62 (SD: 3.41), for the emotions happy, sad, angry, fear and disgust respectively. The mean RTs (ms) for this group were 1431 (SD: 126), 1990 (SD: 157), 1805 (SD: 139), 1840 (SD: 173), 1719 (SD: 138), for the emotions happy, sad, angry, fear and disgust, respectively. We compared the lowest 5\% with the remaining $95 \%$ of scores of EPs and RTs on all emotions (happy, sad, angry, fear and disgust) at preadolescence on the frequency of psychotic experiences at adolescence using independent samples $\mathrm{t}$-tests, finding no significant differences between the groups (see the supplementary table for more information).

\section{Discussion}

Reduced social cognition has often been identified as a trait marker for psychosis, as it is compromised in early phases of psychosis [10], as well as in siblings of individuals diagnosed with a psychotic disorder [13]. We examined whether 


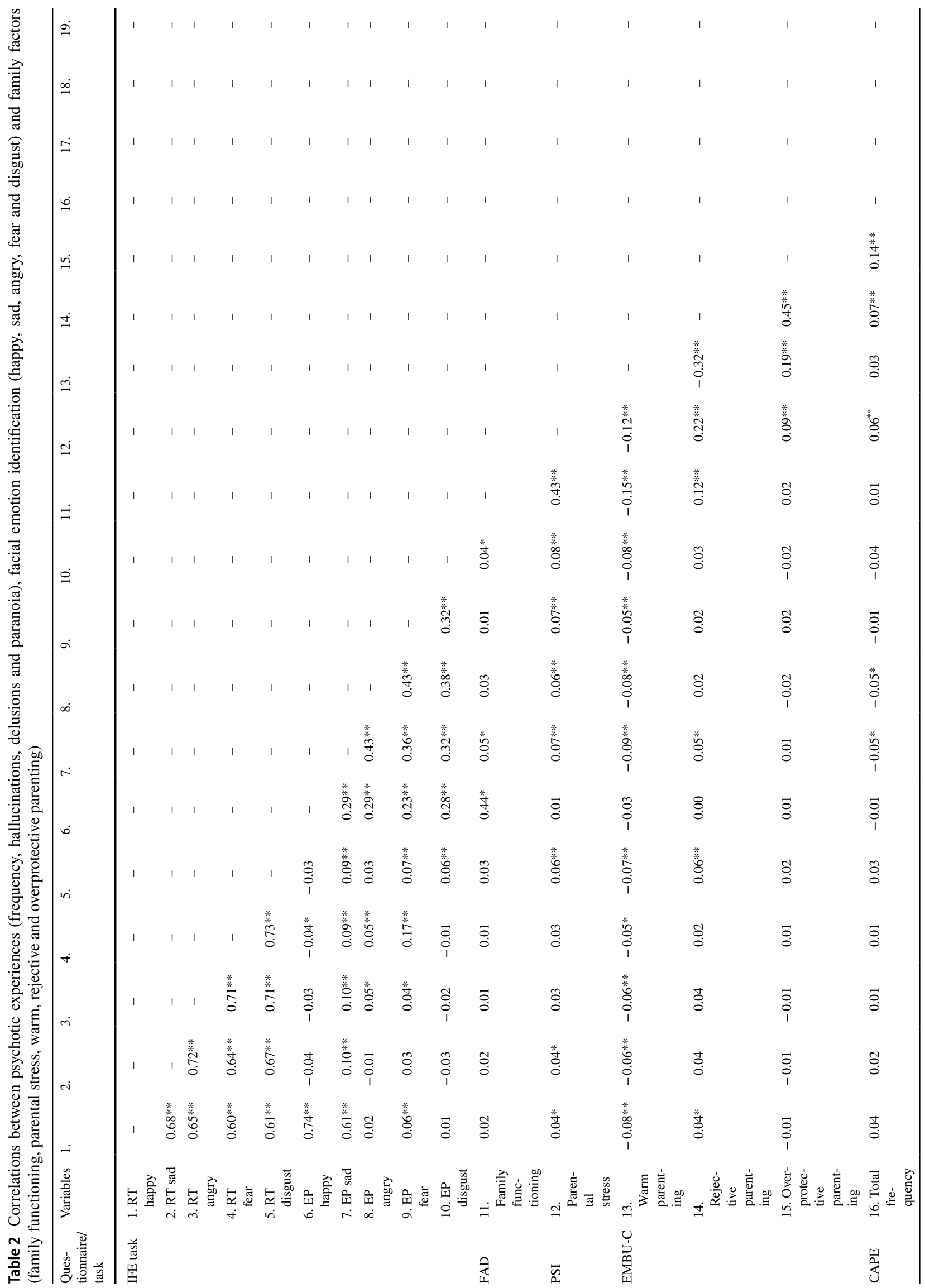




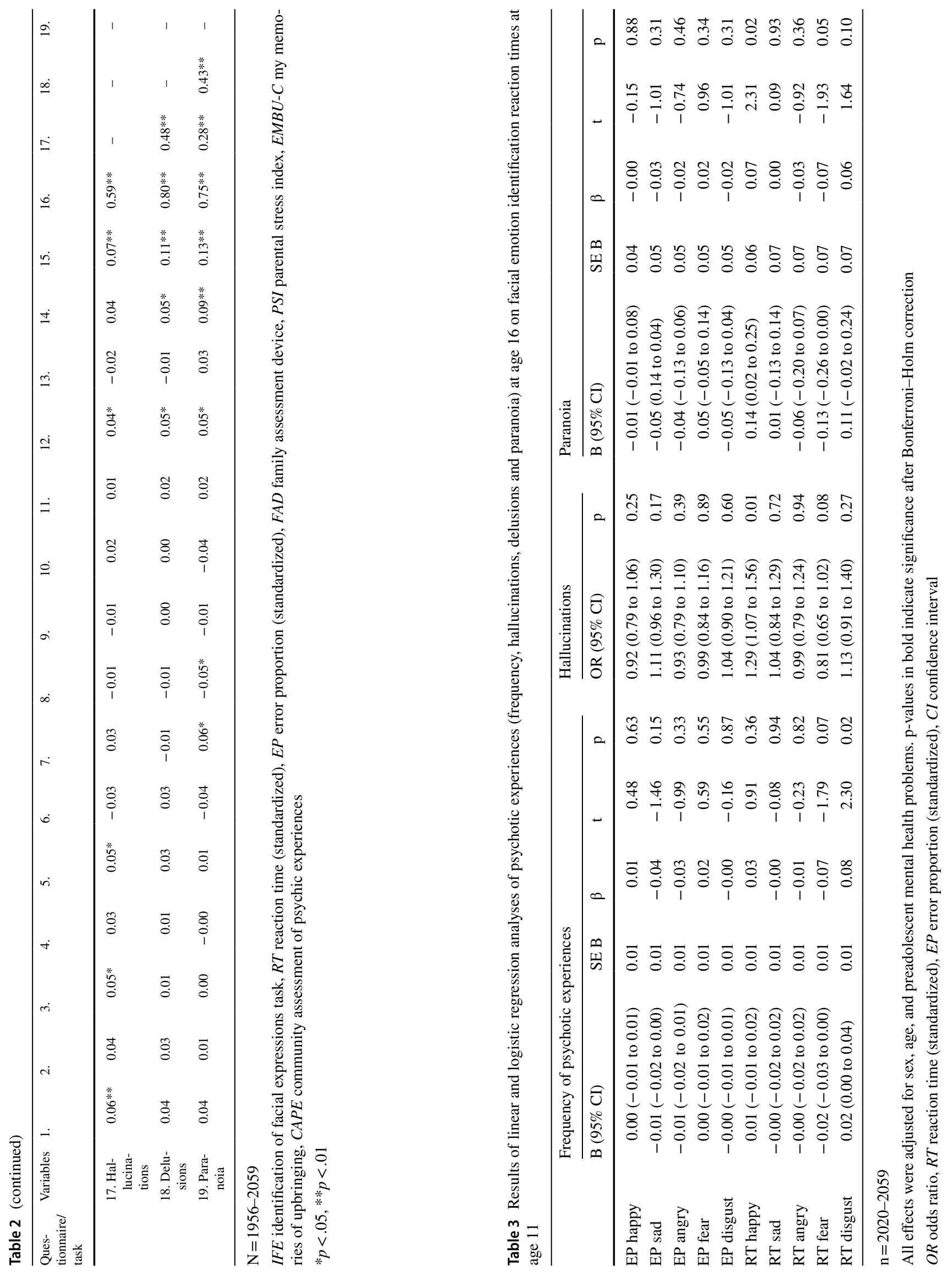


diminished facial emotion identification can be identified as a vulnerability marker for subsequent psychotic experiences in a young adolescent sample. The results did not confirm our hypothesis that facial emotion identification abilities at preadolescence were associated with psychotic experiences at adolescence. When examining a sub-sample of preadolescents scoring the lowest performance on the facial emotion identification task, we still found no vulnerability for psychotic experiences associated with impaired identification of facial affect in adolescence. In absence of an association, mediation by family factors was not explored. As a main effect, increased overprotective parenting at preadolescence was associated with a higher frequency of psychotic experiences as well as delusions in adolescence, after adjustment for preadolescent mental health. There was no indication that parenting stress, family functioning, and rejective and warm parenting were associated with psychotic experiences, indicating these factors may not pose a vulnerability for psychotic experiences.

In the broader adolescent population, when individuals are not recruited for their high risk status or previous episode of psychosis, facial emotion identification does not seem to be predictive of the development of psychotic experiences. Thus, it is possible that the association between facial emotion identification and psychotic experiences is not present in a large and relatively healthy sample. We speculated that perhaps this association would be detectable in a subgroup of adolescents with demonstrably lowered performance in facial emotion identification. However, a post hoc examination based on this subsample also showed no indication of a vulnerability for psychotic experiences over time. Although the reporting of psychotic experiences may increase the risk of developing a mental illness [21, 64-66], the large majority of psychotic experiences are transient and benign during adolescence [67]. Therefore, perhaps an impairment in facial emotion identification is not predictive of psychotic experiences in adolescence, but it may be predictive of clinical psychotic symptoms in young adulthood. This reasoning would be in line with findings of a recent study [68] which reported that developmental cognitive deficits between infancy and adulthood are only found in those who develop a psychotic disorder, with only weak evidence for individuals who have psychotic experiences. The same might hold for the association between facial emotion identification abilities and family functioning, which perhaps becomes evident only at levels of actual impairment.

In the current study, perceived overprotective parenting at preadolescence was predictive of the frequency of psychotic experiences at adolescence, after controlling for early existing mental health problems. It should be noted first that we need to be cautious about the clinical relevance of this finding: the effect of overprotective parenting on the frequency of psychotic experiences was relatively small (denoted by the 


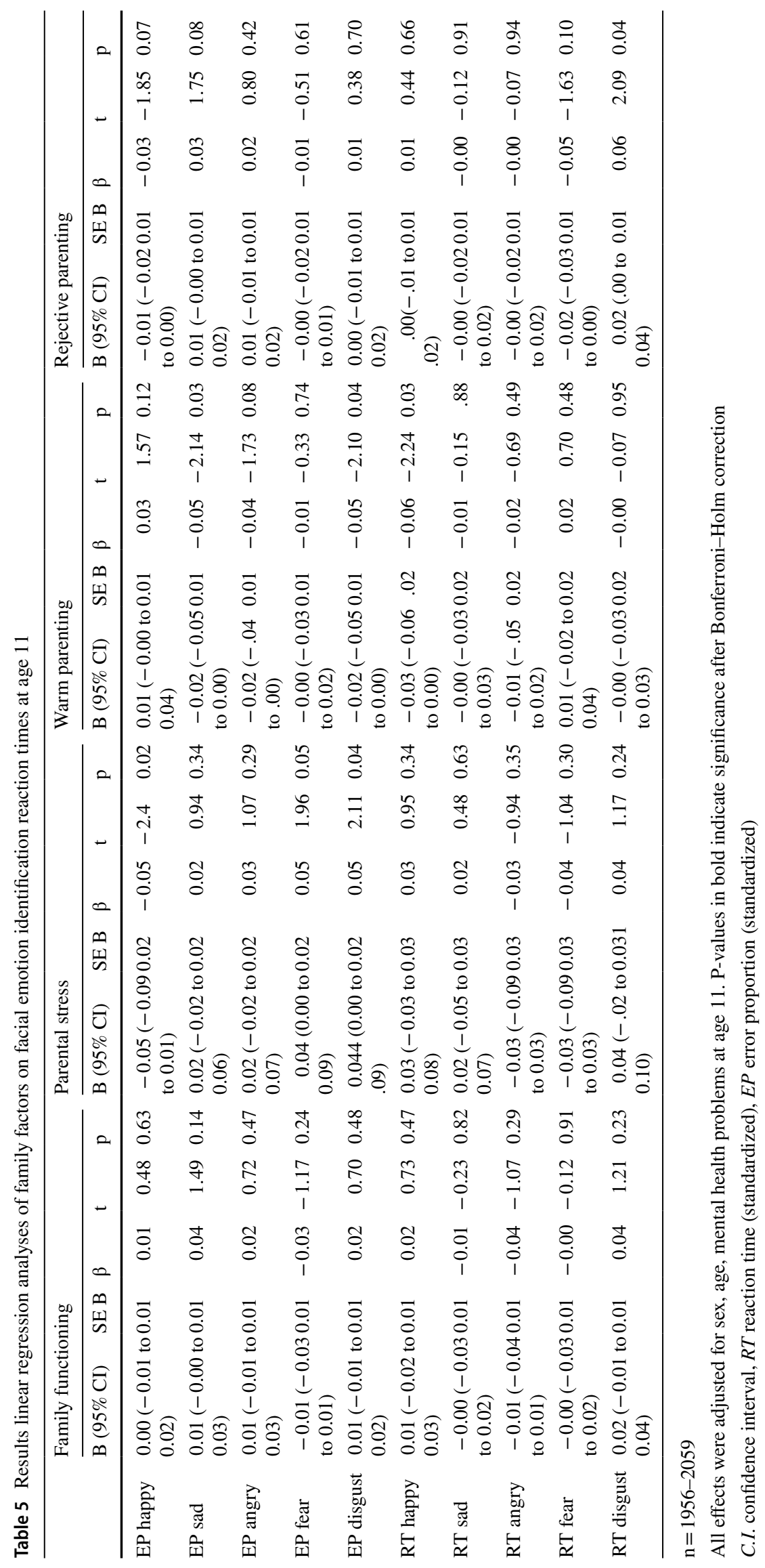


small, but significant correlation and regression coefficient). Second, we need to be cautious about the interpretation. It is possible that when parents overly protect their child, the child is less able to form its own coping mechanisms towards daily stressors. As a result, the child may be less resilient to negative events in life, rendering them more vulnerable to develop psychotic experiences and/or delusions. Another explanation may be that overprotection by the parent is a natural reaction towards a child that is more vulnerable, and requires extra support and care. The parent may sense that the child is sensitive towards certain experiences, and the overprotective parenting may then be an attempt of preventing negative outcomes. However, given that the association was corrected for preadolescent mental health problems, this explanation could be less likely. Overprotective parenting may be a trans-diagnostic risk factor, as previous studies have also found overprotective parenting to be predictive of substance abuse [69, 70], anxiety [71], and internalizing and externalizing problems [72]. Such a risk factor may actually be genetically mediated, which leaves a third explanation that genetic background is causal in both overprotective parenting and in offspring liability to mental health problems. Future research should aim at furthering our understanding of the mechanisms shaping the association.

We expected that rejective parenting, parenting stress, lower family functioning and a lack of warm parenting would also predict psychotic experiences in adolescence, but we did not find evidence for this in the current study. It is possible that overprotective parenting is specifically relevant for the development of psychotic or internalizing problems, whereas rejective parenting may be more relevant for, for example, aggressive problems [73]. An alternative explanation could be that the negative impact of family factors during preadolescence can be compensated with protective factors in adolescence, such as a strong social network of peers. Indeed, previous findings demonstrate that although negative parenting (specifically dominant and harsh parenting) is predictive of externalizing behaviors in adolescence, the association was attenuated by good quality friendships and peer group affiliation [74]. In contrast, overprotective parenting often renders a child placid, cautious and sensitive [75], making them less attractive to peers, and more often at risk of peer victimization [76]. Future research could examine whether the protective effect of peer relationships on negative parenting in preadolescence is less strong (or perhaps not evident) for overprotected children.

This study has a number of limitations. The Facial Expressions Task (ANT [39] is not suited to assess biases in facial emotion identification. An emotional bias is a qualitative deviation in emotional processing [77], such as for example, the under-attribution of happiness when labelling neutral faces [7]. Given that previous studies have found that emotional biases are present and important in psychosis
$[78,79]$ our study would have been more comprehensive to assess biases in addition to the ability to identify emotions per se. In addition, the inclusion of neutral faces would have yielded more information, as processing of neutral faces (a socially ambiguous stimulus) has reported to be abnormal in individuals with a psychotic disorder [80]. A further limitation of our study is the lack of a control group of adolescents with clinical psychotic symptoms, as this would allow us to test our hypothesis that a facial emotion identification vulnerability may only be associated with clinical psychotic symptoms, rather than psychotic experiences more generally. In addition, having knowledge on the family history of mental health problems could shed more light on the potential presence of a genetic liability for both overprotective parenting and psychotic experiences. Last, in the ideal design, we would have assessed psychotic experience at age 11 (rather than general problem behavior), as well as emotion identification at age 16, which would have allowed us to examine concurrent associations that aid in the interpretation of our null findings across these 5 years.

This study also has a number of strengths. First, we used a longitudinal design to examine whether facial emotion identification and family factors would predict psychotic experiences in adolescence, where most studies utilize crosssectional designs (or shorter follow-up periods) and examine these associations in older samples or in samples with individuals who already have psychotic experiences or symptoms, thus limiting the examination of cause-consequence associations. Second, our study has a large sample size and a follow-up period of 5 years. To the best of our knowledge, we were the first to examine in a longitudinal way whether preadolescent facial emotion identification abilities and family factors have the potential to predict psychotic experiences in adolescence.

The current study examined whether facial emotion identification and family factors at preadolescence (age 11) were predictive of psychotic experiences 5 years later at adolescence (age 16). Facial emotion identification at preadolescence was not associated with psychotic experiences at adolescence. This may suggest that a facial emotion recognition vulnerability for psychosis cannot be detected in early adolescence. Alternatively, it may only be evident in subgroups of individuals who ultimately develop a psychotic disorder, indicating that psychotic experiences in adolescence are still too mild or have little specificity for the subsequent psychotic disorder. Overprotective parenting at preadolescence predicted the frequency of both psychotic experiences and delusions, after adjusting for preadolescent mental health. Possibly, overprotective parenting at a young age results in a lack of self-reliance, autonomy or coping skills in adolescents, making them especially vulnerable to psychotic experiences as a reaction to life stressors. However, it could be that overprotection by parents is a natural reaction towards a 
child that is more vulnerable, and requires extra support and care. Likewise, overprotection by parents and their children's vulnerability for psychotic experiences could have a shared background, for example, a shared genetic liability. Future research is needed to examine the mechanism behind the role of overprotective parenting on psychotic experiences during adolescence.

\section{Summary}

An impairment in facial emotion identification could signify a vulnerability for the development of psychosis. Family functioning may mediate the association between facial emotion identification and psychotic experiences. The current study examines whether facial emotion identification and family factors at preadolescence (age 11) predict psychotic experiences 5 years later during adolescence (age 16). Data was obtained from the epidemiological cohort TRAILS (TRacking Adolescents' Individual Lives Survey; N=2059). At preadolescence, a facial emotion identification test and three questionnaires to assess family functioning, perceived parenting styles and parenting stress, were administered. At adolescence, a questionnaire on psychotic experiences was administered. Data were analyzed using multiple linear regression models. Facial emotion identification at preadolescence was not associated with psychotic experiences at adolescence, and the mediational role of family functioning was therefore not further explored. Increased overprotective parenting at preadolescence was associated with a higher frequency of psychotic experiences and delusions at adolescence, while the other family factors (parenting stress, family functioning, and rejective and warm parenting) at preadolescence were not significantly associated with psychotic experiences at adolescence. While clinical symptoms in early and chronic psychosis have been associated with facial emotion identification deficits, this association was not present in the current adolescent cohort. Conversely, perceived overprotective parenting was prospectively associated with psychotic experiences, possibly either due to a vulnerability for psychosis, a natural reaction towards a vulnerable child, or a shared genetic liability in both parents and adolescents. Future research may examine the mechanism behind the role of overprotective parenting on psychotic experiences during adolescence.

Funding This research is funded by the Netherlands Organization for Scientific Research, Grant numbers: GB-MW 940-38-011, 100-001004, 60-60,600-97-118, 261-98-710, GB-MaGW 480-01-006, GBMaGW480-07-001, GB-MaGW 452-04-314, GB-MaGW 452-06-004, 175.010.2003.005,481-08-013, 481-11-001, NWO Vici016.130.002, NWO Gravitation024.001.003; the Ministry of Justice and Security (the Netherlands); European Science Foundation, Grant number: EuroSTRESS pro-ject FP-006; Biobanking and BiomolecularResources Research Infrastructure, Grant number: BBMRI-NL (CP 32); the Gratama foundation; the Jan Dekker foundation; the participating universities; and Accare Centre for Child and Adolescent Psychiatry.

\section{Compliance with Ethical Standards}

Conflicts of interest The authors declare that they have no conflict of interest.

Open Access This article is distributed under the terms of the Creative Commons Attribution 4.0 International License (http://creativeco mmons.org/licenses/by/4.0/), which permits unrestricted use, distribution, and reproduction in any medium, provided you give appropriate credit to the original author(s) and the source, provide a link to the Creative Commons license, and indicate if changes were made.

\section{References}

1. Penn DL, Sanna LJ, Roberts DL (2008) Social cognition in schizophrenia: an overview. Schizophr Bull 34:408-411. https://doi. org/10.1093/schbul/sbn014

2. Green MF, Horan WP (2010) Social Cognition in Schizophrenia. Curr Dir Psychol Sci 19:243-248. https://doi.org/10.1177/09637 21410377600

3. Ekman P (1999) Basic emotions. Handbook of cognition and emotion. Wiley, New York

4. Bediou B, Franck N, Saoud M et al (2005) Effects of emotion and identity on facial affect processing in schizophrenia. Psychiatry Res 133:149-157. https://doi.org/10.1016/j.psychres.2004.08.008

5. Combs DR, Michael CO, Penn DL (2006) Paranoia and emotion perception across the continuum. Br J Clin Psychol 45:19-31. https://doi.org/10.1348/014466505X29099

6. Janssens M, Lataster T, Simons CJP et al (2012) Emotion recognition in psychosis: no evidence for an association with real world social functioning. Schizophr Res 142:116-121. https://doi. org/10.1016/j.schres.2012.10.003

7. Kohler CG, Turner TH, Bilker WB et al (2003) Facial emotion recognition in schizophrenia: intensity effects and error pattern. Am J Psychiatry 160:1768-1774. https://doi.org/10.1176/appi. ajp.160.10.1768

8. Barkl SJ, Lah S, Harris AWF, Williams LM (2014) Facial emotion identification in early-onset and first-episode psychosis: a systematic review with meta-analysis. Schizophr Res 159:62-69. https:// doi.org/10.1016/j.schres.2014.07.049

9. Savla GN, Vella L, Armstrong CC et al (2013) Deficits in domains of social cognition in schizophrenia: a meta-analysis of the empirical evidence. Schizophr Bull 39:979-992. https://doi.org/10.1093/ schbul/sbs080

10. Romero-Ferreiro MV, Aguado L, Rodriguez-Torresano $\mathrm{J}$ et al (2016) Facial affect recognition in early and late-stage schizophrenia patients. Schizophr Res 172:177-183. https://doi. org/10.1016/j.schres.2016.02.010

11. Piskulic D, Liu L, Cadenhead KS et al (2016) Social cognition over time in individuals at clinical high risk for psychosis: findings from the NAPLS-2 cohort. Schizophr Res 171:176-181. https:// doi.org/10.1016/j.schres.2016.01.017

12. Van Donkersgoed RJM, Aleman A, Wunderink L et al (2015) Social cognition in individuals at ultra-high risk for psychosis: a meta-analysis. PLoS ONE 10:e0141075. https://doi.org/10.1371/ journal.pone.0141075 
13. Fett AKJ, Maat A (2013) Social cognitive impairments and psychotic symptoms: what is the nature of their association? Schizophr Bull 39:77-85. https://doi.org/10.1093/schbul/sbr058

14. Pinkham AE, Brensinger $C$, Kohler $C$ et al (2011) Actively paranoid patients with schizophrenia over attribute anger to neutral faces. Schizophr Res 125:174-178. https://doi.org/10.1016/j.schre s.2010.11.006

15. Bentall RP, Kinderman P, Kaney S (1994) The self, attributional processes and abnormal beliefs: towards a model of persecutory delusions. Behav Res Ther 32:331-341. https://doi. org/10.1016/0005-7967(94)90131-7

16. Kohler CG, Bilker W, Hagendoorn M et al (2000) Emotion recognition deficit in schizophrenia: association with symptomatology and cognition. Biol Psychiatry 48:127-136. https://doi. org/10.1016/S0006-3223(00)00847-7

17. Couture SM, Penn DL, Roberts DL (2006) The functional significance of social cognition in schizophrenia: a review. Schizophr Bull 32(Suppl 1):S44-S63. https://doi.org/10.1093/schbul/sbl029

18. Bartels-Velthuis AA, Jenner JA, van de Willige $G$ et al (2010) Prevalence and correlates of auditory vocal hallucinations in middle childhood. Br J Psychiatry 196:41-46. https://doi.org/10.1192/ bjp.bp.109.065953

19. Kelleher I, Connor D, Clarke MC et al (2012) Prevalence of psychotic symptoms in childhood and adolescence: a systematic review and meta-analysis of population-based studies. Psychol Med 42:1857-1863. https://doi.org/10.1017/S0033291711002960

20. Maijer K, Begemann MJH, Palmen SJMC et al (2017) Auditory hallucinations across the lifespan: a systematic review and metaanalysis. Psychol Med. https://doi.org/10.1017/S00332917170023 67

21. Fisher HL, Caspi A, Poulton R et al (2013) Specificity of childhood psychotic symptoms for predicting schizophrenia by 38 years of age: a birth cohort study. Psychol Med 43:2077-2086. https:// doi.org/10.1017/S0033291712003091

22. Brüne $M$ (2005) Emotion recognition, 'theory of mind', and social behavior in schizophrenia. Psychiatry Res 133:135-147. https:// doi.org/10.1016/j.psychres.2004.10.007

23. DuBois DL, Eitel SK, Felner RD (1994) Effects of family environment and parent-child relationships on school adjustment during the transition to early adolescence. J Marriage Fam 56:405-414. https://doi.org/10.2307/353108

24. Butzlaff RL, Hooley JM (1998) Expressed emotion and psychiatric relapse: a meta-analysis. Arch Gen Psychiatry 55:547-552. https://doi.org/10.1001/archpsyc.55.6.547

25. Carter JW, Schulsinger F, Parnas J et al (2002) A multivariate prediction model of schizophrenia. Schizophr Bull 28:649-682. https://doi.org/10.1093/oxfordjournals.schbul.a006971

26. Goldstein MJ (1985) Family factors that antedate the onset of schizophrenia and related disorders: the results of a fifteen year prospective longitudinal study. Acta Psychiatr Scand 71:7-18. https://doi.org/10.1111/j.1600-0447.1985.tb08520.x

27. Hooley JM (2007) Expressed emotion and relapse of psychopathology. Annu Rev Clin Psychol 3:329-352. https://doi. org/10.1146/annurev.clinpsy.2.022305.095236

28. Tienari P, Wynne LC, Sorri A et al (2004) Genotype-environment interaction in schizophrenia-spectrum disorder: long-term followup study of Finnish adoptees. Br J Psychiatry 184:216-222. https ://doi.org/10.1192/bjp.184.3.216

29. Tienari P, Wahlberg KE (2008) Family environment and psychosis. In: Morgan Craig, McKenzie Kwame, Fearon Paul (eds) Society and psychosis. Cambridge University Press, Cambridge

30. Wahlberg KE, Wynne LC, Hakko H et al (2004) Interaction of genetic risk and adoptive parent communication deviance: longitudinal prediction of adoptee psychiatric disorders. Psychol Med 34:1531-1541. https://doi.org/10.1017/S0033291704002661
31. Weintraub MJ, Hall DL, Carbonella JY et al (2017) Integrity of literature on expressed emotion and relapse in patients with schizophrenia verified by a p-curve analysis. Fam Process. https ://doi.org/10.1111/famp.12208

32. Brien MPO, Gordon JL, Bearden CE et al (2006) Positive family environment predicts improvement in symptoms and social functioning among adolescents at imminent risk for onset of psychosis. Schizophr Res 81:269-275. https://doi.org/10.1016/j.schre s.2005.10.005

33. Brien MPO, Zinberg JL, Ho L et al (2009) Family problem solving interactions and 6-month symptomatic and functional outcomes in youth at ultra-high risk for psychosis and with recent onset psychotic symptoms: a longitudinal study. Schizophr Res 107:198-205. https://doi.org/10.1016/j.schres.2008.10.008

34. Marsman R, Oldehinkel AJ, Ormel J, Buitelaar JK (2013) The dopamine receptor $\mathrm{D} 4$ gene and familial loading interact with perceived parenting in predicting externalizing behavior problems in early adolescence: the TRacking adolescents' individual lives survey (TRAILS). Psychiatry Res 209:66-73. https://doi. org/10.1016/j.psychres.2012.10.022

35. Oldehinkel AJ, Rosmalen JGM, Buitelaar JK et al (2015) Cohort profile update: the tracking adolescents' individual lives survey (TRAILS). Int J Epidemiol 44:76. https://doi.org/10.1093/ije/ dyu 225

36. Ormel J, Oldehinkel AJ, Sijtsema J et al (2012) The TRacking adolescents' individual lives survey (TRAILS): design, current status, and selected findings. J Am Acad Child Adolesc Psychiatry 51:1020-1036

37. Zandstra ARE, Hartman CA, Nederhof E et al (2015) Chronic stress and adolescents' mental health: modifying effects of basal cortisol and parental psychiatric history. The TRAILS study. J Abnorm Child Psychol 43:1119-1130. https://doi.org/10.1007/ s10802-014-9970-x

38. De Winter AF, Oldehinkel AJ, Veenstra R et al (2005) Evaluation of non-response bias in mental health determinants and outcomes in a large sample of pre-adolescents. Eur J Epidemiol 20:173-181. https://doi.org/10.1007/s10654-004-4948-6

39. De Sonneville L (1999) Amsterdam neuropsychological tasks: a computer-aided assessment program. In: Den Brinker BPLM et al (eds) Cognitive ergonomics, clinical assessment and computer-assisted learning: computers in psychology. Swets \& Zeitlinger, Lisse

40. Günther T, Herpertz-Dahlmann B, Konrad K (2005) Rehabilitaet von aufmerksamkeits- und verbalen gedaechtnistests bei gesunden kindern und jugendlichen - Implikationen fuer die klinische praxis. Z Kinder Jugendpsychiatr Psychother 33:169-179. https ://doi.org/10.1024/1422-4917.33.3.169

41. Rowbotham I, Pit-ten Cate IM, Sonuga-Barke EJS, Huijbregts SCJ (2009) Cognitive control in adolescents with neurofibromatosis type 1. Neuropsychology 23:50-60. https://doi.org/10.1037/a0013 927

42. Fontaine JRJ, Scherer KR, Roesch EB, Ellsworth PC (2007) The world of emotions is not two-dimensional. Psychol Sci 18:1050 1057. https://doi.org/10.1111/j.1467-9280.2007.02024.x

43. Stevens J (2009) Applied multivariate statistics for the social sciences. Routledge, New York

44. Vrijen C, Hartman CA, Oldehinkel AJ (2016) Slow identification of facial happiness in early adolescence predicts onset of depression during 8 years of follow-up. Eur Child Adolesc Psychiatry 25:1255-1266. https://doi.org/10.1007/s00787-016-0846-1

45. Barkhof E, de Sonneville LMJ, Meijer CJ, de Haan L (2015) Specificity of facial emotion recognition impairments in patients with multi-episode schizophrenia. Schizophr Res 2:12-19. https ://doi.org/10.1016/j.scog.2015.01.001

46. Epstein NB, Baldwin LM, Bishop DS (1983) The McMaster family assessment device *previous first next. J Marital Fam Ther 9:171-180. https://doi.org/10.1111/j.1752-0606.1983.tb01497.x 
47. Miller IW, Epstein NB, Bishop DS, Keitner GI (1985) the McMaster family assessment device: reliability and validity. J Marital Fam Ther 11:345-356. https://doi.org/10.1111/j.1752-0606.1985. tb00028.x

48. Markus MT, Lindhout IE, Boer F et al (2003) Factors of perceived parental rearing styles: the EMBU-C examined in a sample of Dutch primary school children. Personal Individ Differ 34:503 519. https://doi.org/10.1016/S0191-8869(02)00090-9

49. Perris C, Jacobsson L, Linndström H et al (1980) Development of a new inventory for assessing memories of parental rearing behaviour. Acta Psychiatr Scand 61:265-274. https://doi. org/10.1111/j.1600-0447.1980.tb00581.x

50. Bouma EMC, Ormel J, Verhulst FC, Oldehinkel AJ (2008) Stressful life events and depressive problems in early adolescent boys and girls: the influence of parental depression, temperament and family environment. J Affect Disord 105:185-193. https://doi. org/10.1016/j.jad.2007.05.007

51. Kay SR, Wolkenfeld F, Murrill LM (1988) Profiles of aggression among psychiatric patients. I. Nature and prevalence. J Nerv Ment Dis 176:539-546. https://doi.org/10.1097/00005053-19880 9000-00008

52. Oldehinkel AJ, Veenstra R, Ormel J et al (2006) Temperament, parenting, and depressive symptoms in a population sample of preadolescents. J Child Psychol Psychiatry Allied Discip 47:684 695. https://doi.org/10.1111/j.1469-7610.2005.01535.x

53. Abidin RR (1982) Parenting stress and the utilization of pediatric services. Child Health Care 11:70-73. https://doi.org/10.1207/ s15326888chc1102 5

54. Egberink IJL, Frima RM, Vermeulen CSM (1996) COTAN assessment: Nijmeegse Ouderlijke Stress Index (NOSI)

55. Janssens K, Oldehinkel AJ, Rosmalen JGM (2009) Parental overprotection predicts the development of functional somatic symptoms in young adolescents. J Pediatr 154:918-924. https://doi. org/10.1016/j.jpeds.2008.12.023

56. Achenbach TM (1991) Manual for the youth self report and 1991 profile. Univ vermont/department psychiatry

57. Achenbach TM, Dumenci L (2001) Advances in empirically based assessment: Revised cross-informant syndromes and new DSM-oriented scales for the CBCL, YSR, and TRF: Comment on Lengua, Sadowksi, Friedrich, and Fisher (2001). J Consult Clin Psychol

58. Konings M, Bak M, Hanssen M et al (2006) Validity and reliability of the CAPE: a self-report instrument for the measurement of psychotic experiences in the general population. Acta Psychiatr Scand 114:55-61. https://doi.org/10.1111/j.1600-0447.2005.00741.x

59. Peters E, Day S, Mckenna J, Orbach G (1999) Delusional ideation in religious and psychotic populations. Br J Clin Psychol 38:83-96. https://doi.org/10.1348/014466599162683

60. Hanssen M, Peeters F, Krabbendam L et al (2003) How psychotic are individuals with non-psychotic disorders? Soc Psychiatry Psychiatr Epidemiol 38:149-154. https://doi.org/10.1007/s0012 7-003-0622-7

61. Wigman JTW, Vollebergh WAM, Raaijmakers QAW et al (2011) The structure of the extended psychosis phenotype in early adolescence-A cross-sample replication. Schizophr Bull 37:850-860. https://doi.org/10.1093/schbul/sbp154

62. Holm S (1979) A simple sequentially rejective multiple test procedure. Scand J Stat. https://doi.org/10.2307/4615733

63. Hayes AF (2018) Introduction to mediation, moderation, and conditional process analysis: a regression-based approach, 2nd edn. Guilford Press, New York

64. Kaymaz N, Drukker M, Lieb R et al (2012) Do subthreshold psychotic experiences predict clinical outcomes in unselected nonhelp-seeking population-based samples? A systematic review and meta-analysis, enriched with new results. Psychol Med 42:2239-2253
65. Poulton R, Caspi A, Moffitt TE et al (2000) Children's selfreported psychotic symptoms and adult schizophreniform disorder: a 15-year longitudinal study. Arch Gen Psychiatry 57:10531058. https://doi.org/10.1001/archpsyc.57.11.1053

66. Welham J, Scott J, Williams G et al (2009) Emotional and behavioural antecedents of young adults who screen positive for nonaffective psychosis: a 21-year birth cohort study. Psychol Med 39:625-634. https://doi.org/10.1017/S0033291708003760

67. Bartels-Velthuis AA, Wigman JTW, Jenner JA et al (2016) Course of auditory vocal hallucinations in childhood: 11-year follow-up study. Acta Psychiatr Scand 134:1-10. https://doi.org/10.1111/ acps. 12571

68. Mollon J, David AS, Zammit S et al (2018) Course of cognitive development from infancy to early adulthood in the psychosis spectrum. JAMA Psychiatry 75:270-279. https://doi.org/10.1001/ jamapsychiatry.2017.4327

69. Creemers HE, Harakeh Z, Dick DM et al (2011) DRD2 and DRD4 in relation to regular alcohol and cannabis use among adolescents: does parenting modify the impact of genetic vulnerability? The TRAILS study. Drug Alcohol Depend 115:35-42. https://doi. org/10.1016/j.drugalcdep.2010.10.008

70. Visser L, De Winter AF, Vollebergh WAM et al (2013) The impact of parenting styles on adolescent alcohol use: the TRAILS study. Eur Addict Res 19:165-172. https://doi.org/10.1159/000342558

71. Van Oort FVA, Greaves-Lord K, Ormel J et al (2011) Risk indicators of anxiety throughout adolescence: the TRAILS study. Depress Anxiety 28:485-494. https://doi.org/10.1002/da.20818

72. Sentse M, Lindenberg S, Omvlee A et al (2010) Rejection and acceptance across contexts: parents and peers as risks and buffers for early adolescent psychopathology. The TRAILS study. J Abnorm Child Psychol 38:119-130. https://doi.org/10.1007/s1080 2-009-9351-z

73. Sijtsema JJ, Oldehinkel AJ, Veenstra R et al (2014) Effects of structural and dynamic family characteristics on the development of depressive and aggressive problems during adolescence. The TRAILS study. Eur Child Adolesc Psychiatry 23:499-513. https ://doi.org/10.1007/s00787-013-0474-y

74. Lansford JE, Criss MM, Pettit GS et al (2003) Friendship quality, peer group affiliation, and peer antisocial behavior as moderators of the link between negative parenting and adolescent externalizing behavior. J Res Adolesc 13:161-184. https://doi. org/10.1111/1532-7795.1302002

75. Olweus D (1993) Victimization by peers: antecedents and longterm outcomes. In: Rubin K, Aseodorf JB (eds) Social withdrawal, inhibition, and shyness in childhood. University of Chicago Press, Chicago, pp 315-341

76. Smith PK, Myron-Wilson R (1998) Parenting and school bullying. Clin. Child Psychol. Psychiatry 3:405-417

77. Dondaine T, Robert G, Péron J et al (2014) Biases in facial and vocal emotion recognition in chronic schizophrenia. Front Psychol. https://doi.org/10.3389/fpsyg.2014.00900

78. Weiss EM, Kohler CG, Brensinger CM et al (2007) Gender differences in facial emotion recognition in persons with chronic schizophrenia. Eur Psychiatry 22:116-122. https://doi.org/10.1016/j. eurpsy.2006.05.003

79. Premkumar P, Cooke MA, Fannon D et al (2008) Misattribution bias of threat-related facial expressions is related to a longer duration of illness and poor executive function in schizophrenia and schizoaffective disorder. Eur Psychiatry 23:14-19. https://doi. org/10.1016/j.eurpsy.2007.10.004

80. Derntl B, Habel U (2017) Angry but not neutral faces facilitate response inhibition in schizophrenia patients. Eur Arch Psychiatry Clin Neurosci. https://doi.org/10.1007/s00406-016-0748-8

Publisher's Note Springer Nature remains neutral with regard to jurisdictional claims in published maps and institutional affiliations. 